${ }^{7}$ L. Pauling and E. B. Wilson, Introduction to Quantum Mechanics (McGraw-HIll, New York, 1935), p. 144.

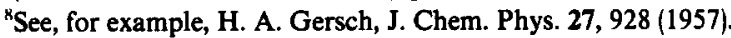

${ }^{9}$ E. Jahnke and F. Emde, Tables of Functions (Dover, New York, 1945), p. 143

${ }^{10}$ This expression for the root-mean-squared amplitude is seen to follow from the virtual theorem $\langle E\rangle=2\langle V\rangle=m \omega^{2}\left\langle x^{2}\right\rangle$ and $\langle E\rangle=E_{n^{\prime}}=\left(n^{\prime}+1 / 2\right) \hbar \omega$.

"F. T. Hioie and E. W. Montroll, J. Math. Phys. 16, 1945 (1975).

${ }^{12}$ C. M. Bender, F. Cooper, G. S. Guralnik, and D. S. Sharp, Phys. Rev. D $19,1865(1979)$.

\title{
Influence of air drag on the optimal hand launching of a small, round projectile
}

\author{
J. A. Zufiria and J. R. Sanmartín \\ Escuela Técnica Superior de Ingenieros Aeronáuticos, Universidad Politécnica de Madrid, Madrid, Spain
}

(Received 3 January 1980; accepted for publication 4 March 1981)

Conditions leading to a maximum range for a small, round projectile, fired by hand, are discussed taking into account air drag and the dependence of the initial speed on the mass launched. Both the optimal angle of release for given projectile and initial speed, and the optimal radius for given density (i.e., among a bed of pebbles) are determined; an increase on the height of release is found to always decrease the angle and increase the radius. The influence of the projectile mass on the optimal manner of launching is considered. The validity of the approximations used in the analysis is discussed. Results from very simple measurements show good agreement with theory.

\section{INTRODUCTION}

The motion of a small, round, projectile, e.g., a stone, through air, under the action of gravity, is a standard problem in point dynamics.' For a broad range of sizes and speeds, the air drag is proportional to the square of the speed and the entire motion may be analytically determined in terms of single integrals.

On the other hand, the practical (?) problem confronted by a person who wishes to maximize the horizontal range of a hand throw, when parameters such as size and density of the projectile, or angle and way of throwing, are considered, seems to have been overlooked, except for the limiting case of negligible air drag, recently discussed by Lichtenberg and Willis. ${ }^{2-4}$ The purpose of this paper is to establish criteria of optimization of those parameters. The problem that motivated the study initially, was the one a child solves often when, from a bed of pebbles, he selects a particular one to hurl.

\section{STATEMENT OF THE PROBLEM}

We consider a sphere of radius $R$, density $\rho$, and mass $m \equiv 4 \pi R^{3} \rho / 3$, launched from a point at height $h$ over level ground, at speed $v_{0}$ and angle $\alpha$ above the horizontal. We assume that the sphere has no spin and that the Reynolds number, $R_{e}=2 R \rho_{a} v / \mu_{a}$, satisfies throughout the motion the condition

$$
10^{3}<R_{e} \leqslant 10^{5}
$$

the air drag may then be written as

$$
F_{D}=C_{D} \frac{1}{2} \rho_{a} v^{2} \pi R^{2}(-\bar{v} / v) \text {, }
$$

where $C_{D}=0.45$ within a $10 \%$ error, ${ }^{5} \rho_{a}$ and $\mu_{a}$ are the air density and viscosity, respectively, and $\bar{v}$ is the velocity. Equation (2), established for constant $\bar{v}$, will remain clearly valid for conditions such that the characteristic time for variations of $\bar{v}$ is large compared with the time for the sphere to cross a characteristic length of the flow field, that is,

$$
v /\langle|d \bar{v} / d t|\rangle\rangle R / v \text {. }
$$

We shall later comment on the restrictions (1) and (3).

From the equation of motion

$$
m \frac{d \bar{v}}{d t}=m \bar{g}-\frac{1}{2} C_{D} \rho_{a} \pi R^{2} v \bar{v},
$$

and taking the $y$ axis.upwards, we immediately obtain

$$
\frac{d v_{y}}{d v_{x}}=\frac{v_{*}^{2}+v_{y}\left(v_{x}^{2}+v_{y}^{2}\right)^{1 / 2}}{v_{x}\left(v_{x}^{2}+v_{y}^{2}\right)^{1 / 2}},
$$

where

$$
v_{*}^{2} \equiv \frac{m g}{C_{D} \frac{1}{2} \rho_{a} \pi R^{2}}=\frac{8 g R \rho}{3 C_{D} \rho_{a}} ;
$$

the ratio

$$
\sigma \equiv v_{*}^{2} / v_{0}^{2}
$$

is a parameter measuring the relative importance of the weight against the air drag. Defining

$$
\begin{aligned}
& \beta=-v_{y} / v_{x}, \quad[p \equiv \tan \alpha=-\beta(t=0)], \\
& F(\beta)=\operatorname{arcsinh} \beta+\beta\left(1+\beta^{2}\right)^{1 / 2},
\end{aligned}
$$

Eq. (4) yields a standard result ${ }^{1}$

$$
v_{x}^{2}=v_{0}^{2}\left(1+p^{2}+\frac{F(p)+F(\beta)}{\sigma}\right)^{-1} \equiv v_{0}^{2} G(p, \sigma, \beta) .
$$

Using

$$
d x=g^{-1} v_{x}^{2} d \beta, \quad d y=-g^{-1} v_{x}^{2} \beta d \beta,
$$

we finally get

$$
\begin{aligned}
& \frac{g L}{v_{0}^{2}}=\int_{-p}^{\beta_{f}} G(p, \sigma, \beta) d \beta, \\
& \frac{g h}{v_{0}^{2}}=\int_{-p}^{\beta_{f}} G(p, \sigma, \beta) \beta d \beta,
\end{aligned}
$$


yielding $g L / v_{0}^{2}$ as a function of $p, \sigma$, and $g h / v_{0}^{2} ; \beta_{f}(p, \sigma$, $g h / v_{0}^{2}$ ) is the final value of $\beta$.

Our purpose is to maximize $L$, which is a function of $v_{0}$, $p, R, \rho$, and $h$. Most often $v_{0}$ will not be considered an independent parameter. In this respect, we shall assume that a given person launching a projectile in a certain way may be characterized by just two quantities: an effective mass $M_{e}$ for the part of the body set in motion in the throw, and the kinetic energy $E$ imparted to it and to the projectile, both $M_{e}$ and $E$ being independent of $m$. We then write

$$
v_{0}=\left[2 E /\left(m+M_{e}\right)\right]^{1 / 2} \text {. }
$$

$M_{\mathrm{e}}$ is clearly less than the mass set in motion (only the hand has the speed $v_{0}$ at the time of release) and may be estimated from geometrical considerations: for instance, if only the arm, assumed cylindrical, is moved, $M_{e}$ is one third of its mass. Instead of $E$, for convenience, we shall use the limiting value of $v_{0}$ as $m \rightarrow 0$

$$
v_{1} \equiv\left(2 E / M_{e}\right)^{1 / 2}
$$

which may be measured easily.

The preceding model is based on the hypotheses that, in a throw, muscles develop their maximum force independently of the value of $m$ (which affects the speed of muscle contraction), and that the ratio $\eta$ of (E/force) to muscle length is also independent of $m$; then $E$ is independent too ( $M_{e}$ is clearly so). The model allows to estimate $v_{1}$. Introducing the maximum force per unit area of muscle fiber $T_{a}$ (about $20 \mathrm{~N} \mathrm{~cm}^{-2}$ typically ${ }^{6}$ ), the muscle density $\rho_{\mathrm{mu}}$ (about $1 \mathrm{~g} \mathrm{~cm}^{-3}$ ), and the muscle mass $M_{\text {mu }}$, we have

$$
v_{1}=\left(\frac{2 T_{a}}{\rho_{\mathrm{mu}}} \eta \frac{M_{\mathrm{mu}}}{M_{e}}\right)^{1 / 2} \simeq 20 \eta^{1 / 2}\left(\frac{M_{e}}{M_{\mathrm{mu}}}\right)^{1 / 2} \mathrm{~m} / \mathrm{sec}
$$

For $\eta$ somewhat less than unity and $\left(M_{m u} / M_{e}\right)^{1 / 2}$ near unity, we may roughly write

$$
15<v_{1}(\mathrm{~m} / \mathrm{sec})<25 \text {. }
$$

The influence of the height $h$ on $L$ is weak for a reasonable thrower. By this we mean that "his" value of $g h / v_{1}^{2}$ is small (typically about 0.05 ) and that he only considers $p$ 's of order of unity (the value of $p$ maximizing $L, p_{\text {opt }}$, is of order of unity under all acceptable conditions, as we shall see). Then if $m / M_{e}$ is not too large, $g h / v_{0}^{2}$ will be small and will have little effect on the value of $L$ (this is not the case, obviously, if $p$ is small). In Sec. III, to maximize the range, we first consider a vanishing height of release, for which we have

$$
\begin{aligned}
& g L / v_{0}^{2}=\int_{-p}^{\beta_{f 0}} G(p, \sigma, \beta) d \beta \equiv I(p, \sigma), \\
& 0=\int_{-p}^{\beta_{f 0}} G(p, \sigma, \beta) \beta d \beta \quad \beta_{f 0} \equiv \beta_{f}(h=0),
\end{aligned}
$$

and then briefly comment on the small $g h / v_{1}^{2}$ corrections.

\section{PARAMETER OPTIMIZATION}

\section{A. Optimal angle}

Figure 1 shows $I\left(\alpha \equiv \tan ^{-1} p, \sigma\right)$. The optimal angle depends only on $\sigma$ and is found by setting $\partial I / \partial \alpha=0$. Both $\alpha_{\mathrm{opt}}(\sigma)$ and $I_{\max }(\sigma) \equiv I\left[\alpha_{\mathrm{opt}}(\sigma), \sigma\right]$ are given in Fig. 2; then

$$
L\left(\alpha_{\text {opt }}\right)=v_{0}^{2} g^{-1} I_{\max }(\sigma) \text {. }
$$

For $\sigma \rightarrow \infty$ we recover the zero-drag results

$$
I \rightarrow \sin 2 \alpha, \quad \alpha_{\text {opt }} \rightarrow \pi / 4, \quad I_{\max } \rightarrow 1 .
$$

For $\sigma \rightarrow 0$ both $\alpha_{\mathrm{opt}}$ and $I_{\max }$ vanish; $\alpha_{\mathrm{opt}}$ behaves roughly as $\left(\ln \sigma^{-1}\right)^{-1}$ so that $\alpha_{\text {opt }}$ will not be small unless $\sigma$ is extremely small, and then our analysis breaks down (see Sec. IV). Notice that $I(\alpha, \sigma) \simeq I_{\max }(\sigma)$ for a broad range of angles.

\section{B. Optimal radius}

Further optimization is possible if the characteristic values $v_{1}$ and $M_{e}$ of the thrower, and the density $\rho$, are given but the radius $R$ is not, as in the problem of determining the optimal size of a stone for a hand throw. We write

$$
\begin{gathered}
L\left(\alpha_{\mathrm{opt}}\right)=\frac{v_{1}^{2}}{g} \frac{I_{\max }(\sigma)}{1+m / M_{e}}, \quad \sigma=A\left(1+m / M_{e}\right)\left(m / M_{e}\right)^{1 / 3}, \\
A \equiv\left(128 / 9 \pi C_{D}^{3}\right)^{1 / 3}\left(g / \rho_{a}\right) \rho^{2 / 3} M_{e}^{1 / 3} v_{1}^{-2} .
\end{gathered}
$$

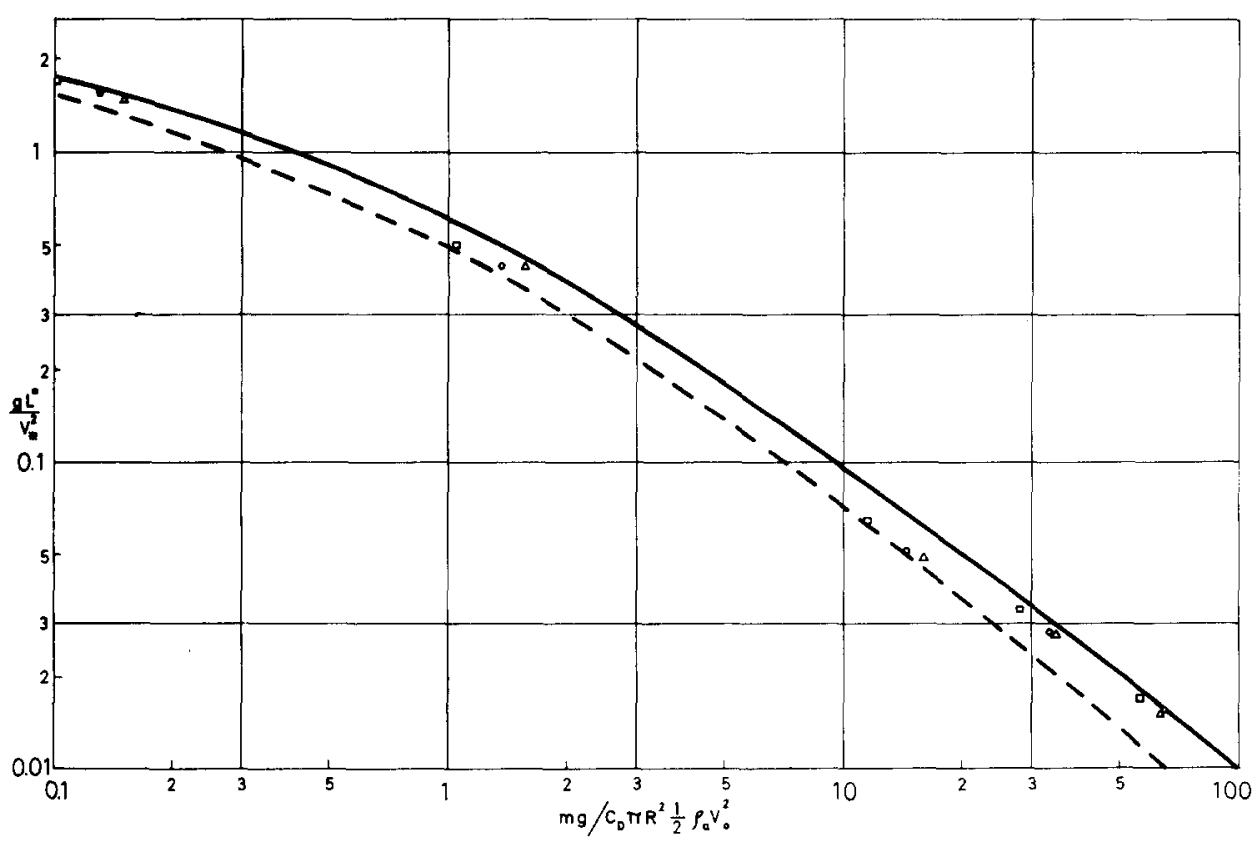

60 Am. J. Phys., Vol. 50, No. 1, January 1982
Fig. 1. Dimensionless range versus weight to drag initial ratio $\sigma$, and angle of release, for zero height of release. 


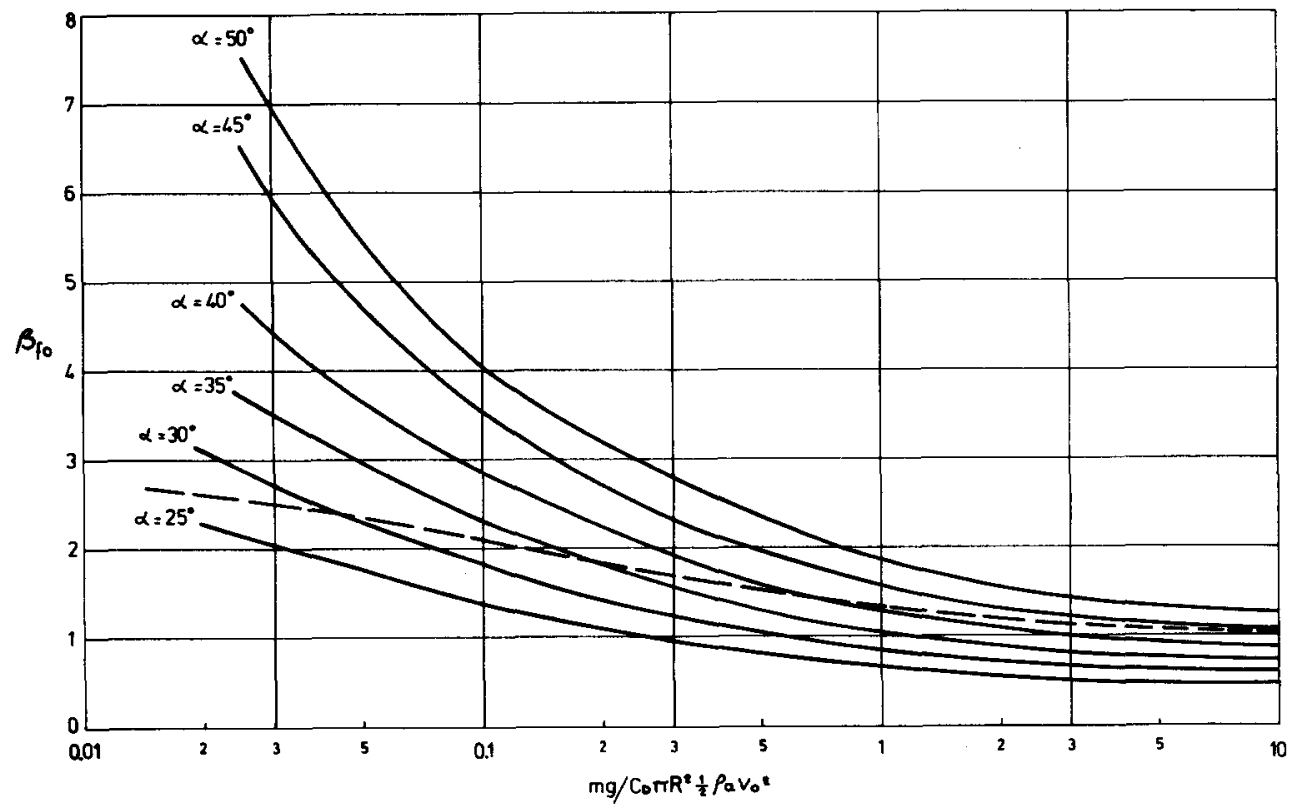

Fig. 2. Optimal angle of release and maximum range, versus weight to drag initial ratio $\sigma$, for zero height of release.

For any given $A,\left(1+m / M_{e}\right)^{-1} I_{\max }$ is a function of $R\left(M_{e}\right)$ $\rho)^{-1 / 3}$ and presents a maximum at an optimal value $R_{\mathrm{op}}$, shown with its range in Fig. 3. For $A$ large the maximum is too broad to properly speak of one optimal value; this is indicated in Fig. 3.

\section{Optimal density}

Similarly, if $v_{1}, M_{e}$, and $R$ are given and $\rho$ is not, we write

$$
\begin{aligned}
& L\left(\alpha_{\mathrm{opt}}\right)=\frac{v_{1}^{2}}{g} \frac{I_{\max }(\sigma)}{1+m / M_{e}}, \quad \sigma=B\left(1^{\prime}+m / M_{e}\right) m / M_{e}, \\
& B \equiv\left(2 / \pi C_{D}\right)\left(g / \rho_{a}\right) R^{-2} v_{1}^{-2} M_{e} .
\end{aligned}
$$

For any given $B,\left(1+m / M_{e}\right)^{-1} I_{\max }$ is a function of $\left(R^{3} / M_{e}\right) \rho$ and presents a maximum at an optimal value $\rho_{\text {opt }}$, shown with its range in Fig. 4. Again for large $B$ the maximum is too broad to be significant.

\section{Other optimizations}

If $v_{1}$ and $M_{e}$ are given and the projectile $(\rho, R)$ is not, we write

$$
L\left(\alpha_{\mathrm{opt}}\right)=\frac{v_{1}^{2}}{g} \frac{I_{\max }(\sigma)}{1+m / \boldsymbol{M}_{e}}, \quad \sigma=\left(1+m / M_{e}\right) v_{*}^{2} / v_{1}^{2} .
$$

To maximize $L\left(\alpha_{\text {opt }}\right)$ it will suffice to choose $m^{-1}$ and $v_{*}^{2}$ so large that $I_{\max } \simeq 1,1+m / M_{e} \simeq 1$. That implies taking $\rho$ and $R^{-1}$ large; more explicitly

$$
\rho R^{3}<M_{e}, \quad \rho R>\rho_{a} v_{1}^{2} / g .
$$

Then the air drag is negligible.

If the projectile is given but the way of throwing is not, we write

$$
L\left(\alpha_{\mathrm{opt}}\right)=\frac{v_{*}^{2}}{g} \frac{I_{\max }(\sigma)}{\sigma}, \quad \sigma=\left(1+m / M_{e}\right) v_{*}^{2} / v_{1}^{2} .
$$

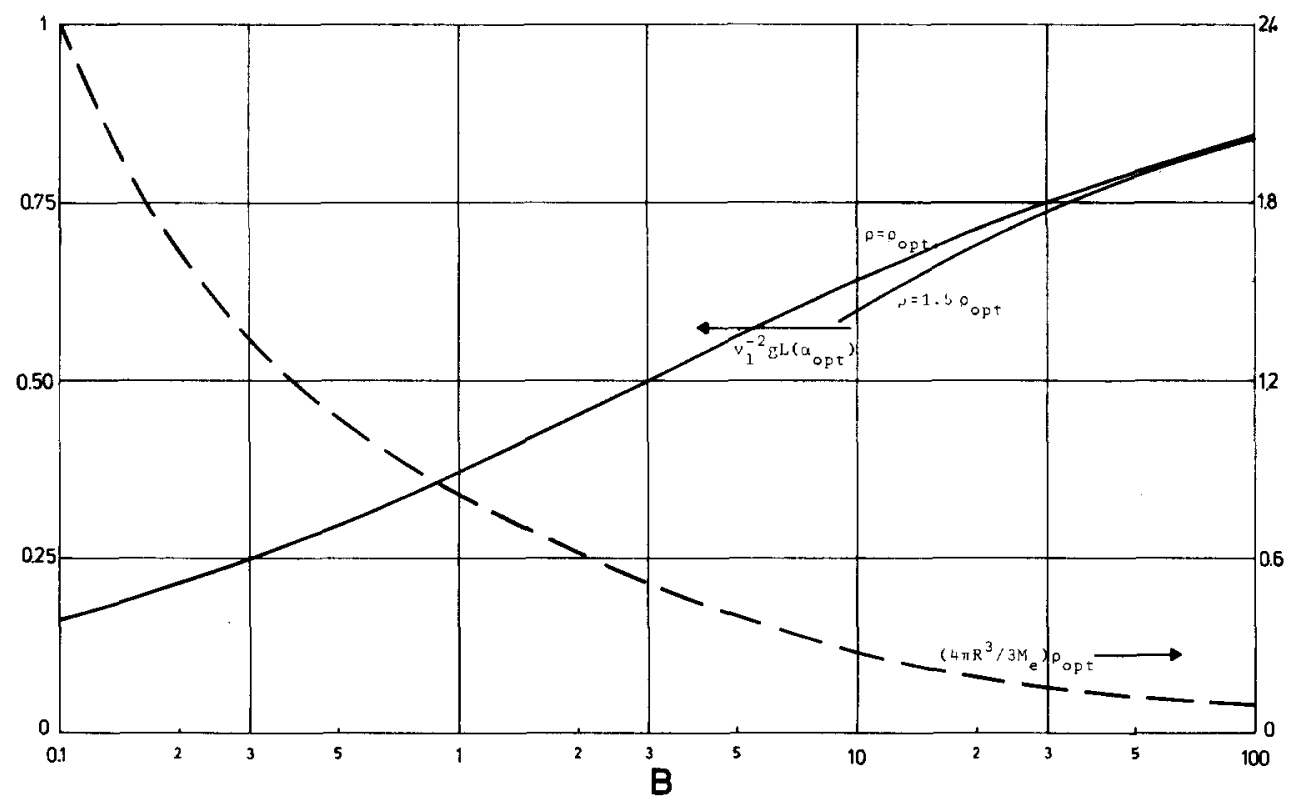

Fig. 3. Dimensionless range $v_{1}^{-2} g L\left(\alpha_{\mathrm{opt}}\right) \quad \longrightarrow$ for $R=R_{\mathrm{opt}}$ and $R=1.5 R_{\mathrm{opt}}$, and optimal radius $\left(4 \pi p / 3 M_{e}\right)^{1 / 3} R_{\text {opt }}(---)$, versus the parameter $A$ [Eq. (11)]. 


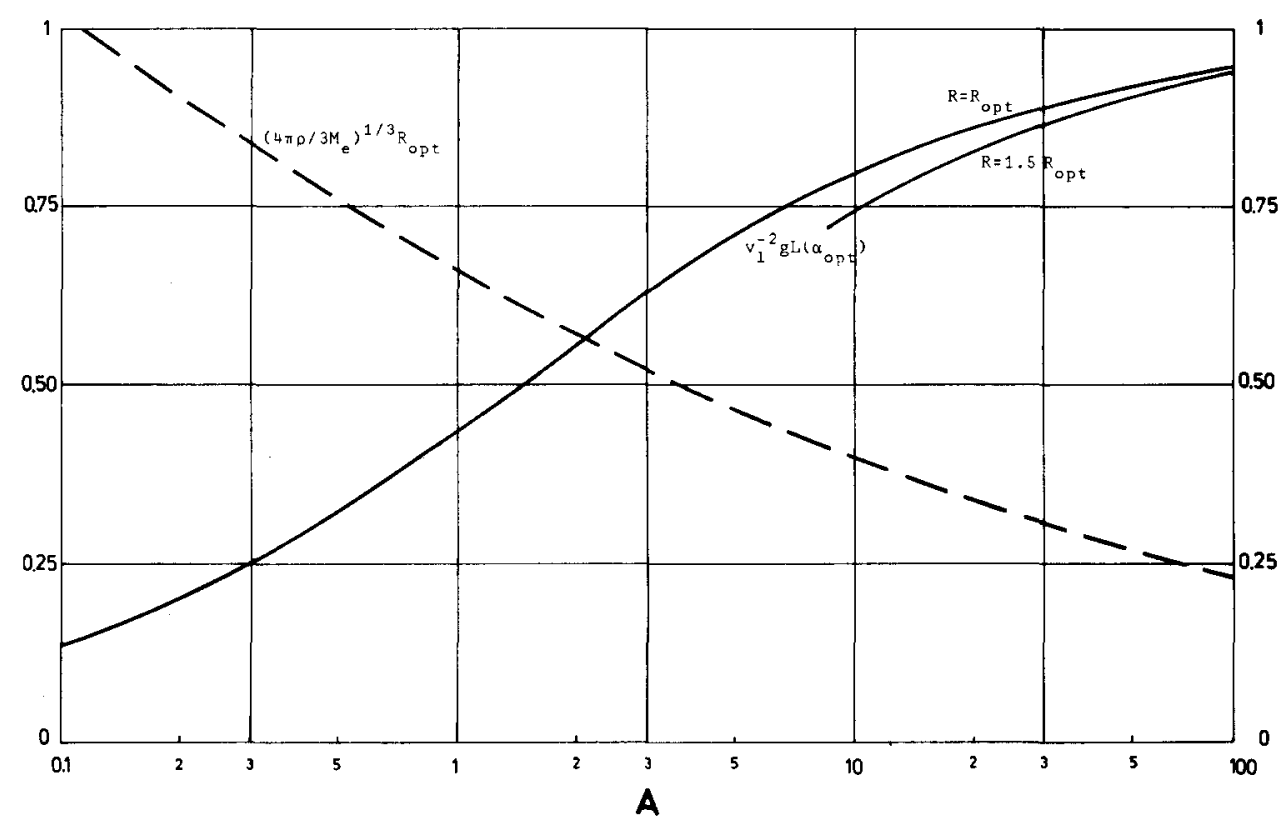

Fig. 4. Dimensionless range $v_{1}^{-2}$ $g L\left(\alpha_{\mathrm{opt}}\right)(\longrightarrow)$ for $\rho=\rho_{\mathrm{opt}}$ and $\rho=1.5 \rho_{\text {opt }}$, and optimal density $\left(4 \rho \pi R^{3} / 3 M_{e}\right) \rho_{\text {opt }}(---)$, versus the parameter $B$ [Eq. (12)].

Since $I_{\max }(\sigma) / \sigma$ decreases monotonically as $\sigma$ grows, optimization trivially reduces to choosing $v_{0}^{2}=\left(1+m / M_{e}\right)^{-1}$ $v_{1}^{2}$, for fixed $m$, as large as possible. Notice, however, that then one chooses dominant drag conditions.

\section{E. Small $g h / v_{l}^{2}$ corrections}

For a nonvanishing height $h$ we have, from (7)-(10),

$$
\begin{aligned}
& L=v_{0}^{2} g^{-1} I(\alpha, \sigma)+\Delta L \\
& g \Delta L / v_{0}^{2}=\int_{\beta_{f o}}^{\beta_{f}} G(p, \sigma, \beta) d \beta, \\
& g h / v_{0}^{2}=\int_{\beta_{f o}}^{\beta_{j}} G(p, \sigma, \beta) \beta d \beta .
\end{aligned}
$$

Since $g \Delta L / v_{0}^{2} I$ increases with $\sigma$ we may consider $\sigma \geqslant 0(1)$; then $\beta_{f 0}=0(1)$ as shown in Fig. 5. It follows from (14) that

$$
\Delta L \simeq h / \beta_{f 0}(\alpha, \sigma)
$$

An expression also valid for $\sigma \ll 1$ is $\Delta L=\left(v_{0}^{2} \sigma / g \beta_{f 0}\right)$ $\left[1-\exp \left(-g h / v_{0}^{2} \sigma\right)\right]$.
From (13) and (15) we find the small correction to the optimal angle

$$
\Delta \alpha_{\mathrm{opt}}=-\left.\frac{g h}{v_{0}^{2}} \frac{\partial \beta_{f 0}^{-1} / \partial \alpha}{\partial^{2} I / \partial \alpha^{2}}\right|_{\alpha_{\mathrm{cpt}}}<0
$$

and the correction $h / \beta_{\text {opt }}(\sigma)$ to the maximum range; here $\beta_{\mathrm{opt}}(\sigma) \equiv \beta_{f 0}\left[\alpha_{\mathrm{opt}}(\sigma), \sigma\right]$, given in Fig. 5 too. We also find $\Delta R_{\mathrm{opt}}>0, \Delta \rho_{\mathrm{opt}}>0$.

\section{DISCUSSION}

We have studied how to maximize the horizontal range $L$ of a small, round projectile of density $\rho$ and radius $R$, thrown by hand. The dependence of the initial speed $v_{0}$ on the projectile mass $m$ was assumed to be $v_{0}=v_{1}\left(1+m / M_{e}\right)^{-1 / 2}$, where $v_{1}$ and $M_{e}$ are a limiting velocity and an effective mass that can be determined for any given person with a particular way of throwing.

For fixed $\rho, R, M_{e}$, and $v_{1}$ there is an optimal angle of release $\alpha_{\text {opt }}$, function of the weight to air drag initial ratio $\sigma \equiv 4.5 \times 10^{2} R(\mathrm{~cm}) \rho\left(\mathrm{g} / \mathrm{cm}^{3}\right)\left[v_{0}(\mathrm{~m} / \mathrm{sec})\right]^{-2} ; \alpha_{\text {opt }}$ and

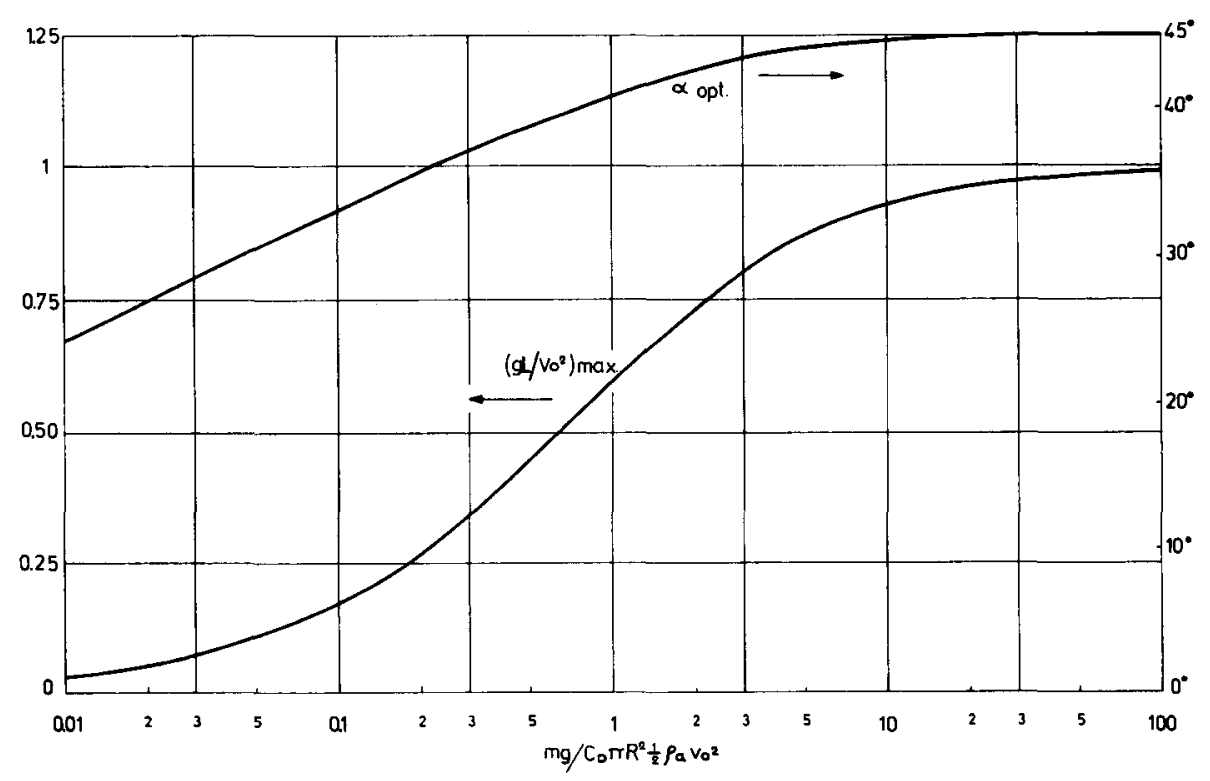

Fig. 5. Slope $\beta_{f o}$ of the trajectory when reaching the height of release, versus weight to drag initial ratio $\sigma$, and angle of release. Also shown is $\beta_{f 0}$ for the optimal $\alpha$. 
$L\left(\alpha_{\text {opt }}\right)$ are shown in Fig. 2. For fixed $\rho, M_{e}$, and $v_{1}$, there is an optimal radius $R_{\text {opt }}$ shown in Fig. 3, together with $L\left(\alpha_{\mathrm{opt}}, R_{\mathrm{opt}}\right)$, as a function of the parameter $A \simeq 2.8 \times 10^{3}$ $\left[\rho\left(\mathrm{g} / \mathrm{cm}^{3}\right)\right]^{2 / 3}\left[M_{e}(\mathrm{~kg})\right]^{1 / 3}\left[v_{1}(\mathrm{~m} / \mathrm{sec})\right]^{-2}$; this result solves the problem mentioned in Sec. I: to choose, from a bed of pebbles, that one maximizing $L$. For fixed $R, M_{e}$, and $v_{1}$, there is an optimal density $\rho_{\mathrm{opt}}$ shown in Fig. 4, together with $L\left(\alpha_{\text {opt }}, \rho_{\text {opt }}\right)$, as function of the parameter $B \simeq 1.07 \times 10^{5} M_{e}(\mathrm{~kg})\left[R(\mathrm{~cm}) v_{1}(\mathrm{~m} / \mathrm{sec})\right]^{-2}$. These results corespond to zero height of release $h$. Corrections due to a nonvanishing $h$ are of order of $g h / v_{0}^{2}$, a small quantity if $m / M_{e}$ is not large $\left(g h / v_{1}^{2} \simeq 0.05\right.$ typically). The correction to $\alpha_{\mathrm{opt}}$ is a decrease, as in the known limit of zero air $\mathrm{drag}^{2}$; on the other hand, $R_{\text {opt }}$ and $\rho_{\text {opt }}$ increase with $h$.

For a given projectile $(\rho, R), L$ is maximum for $v_{0}$ maximum; to maximize $v_{0}$ a person may choose among two or three couples of values $M_{e}$ and $v_{1}$. For $m$ small enough it will suffice to choose the maximum $v_{1}$ because $v_{0} \simeq v_{1}$ in all cases; this suggests that $v_{1}$ is largest for the way of hurling that people use for small stones, for which $\boldsymbol{M}_{e} \simeq 1 \mathrm{~kg}$. On the other hand, for the shot put $m(\simeq 7.25 \mathrm{~kg})$ is so large that it proves convenient to take a smaller $v_{1}$ if the value of $M_{e}$ is large enough (of the order of $20 \mathrm{~kg}$ for the way used in shot putting).

Our analysis was based on conditions (1) and (3). The last one, requiring both $v^{2}>g R$ and $m v^{2} / R>\frac{1}{2} C_{D} \rho_{a} \pi R^{2} v^{2}$ (that is, $6 \rho>\rho_{a}$ ), is clearly satisfied unless $\rho$ is about the air density, and then buoyancy effects should have been considered anyway. Condition (1) may be written $0.75<R$ $(\mathrm{cm}) v(\mathrm{~m} / \mathrm{sec}) \leqslant 75$. The upper bound may be exceeded if $R$ is large enough: for the shot put $R \simeq 6 \mathrm{~cm}$ and the condition is marginally satisfied $\left[v_{0} \simeq 15 \mathrm{~m} / \mathrm{sec}\right.$ typically, and $\left.R(\mathrm{~cm}) v_{0}(\mathrm{~m} / \mathrm{sec}) \simeq 90\right]$; in fact, for such large radii and not low densities, $\sigma$ is so large that the air drag, and thus the Reynolds number influence, is negligible. At the other end, only radii too small to be of interest can get $R v$ below its lower bound $(R<0.04 \mathrm{~cm}$ if $v \simeq 20 \mathrm{~m} / \mathrm{sec})$. For $R>0.04$ $\mathrm{cm}, \rho>10 \rho_{a}$, and $v_{0} \simeq 20 \mathrm{~m} / \mathrm{sec}$, we have $\sigma>10^{-3}$, and then $\alpha_{\text {opt }}=0(1)$ as noticed in Sec. III.

To check our model experimentally we carried out easy measurements of $R, m$, and $L$; the weak dependence of $L$ on
Table I. Projectile characteristics.

\begin{tabular}{lllll}
\hline \hline Projectiles & $\begin{array}{l}\text { Density } \\
\rho\left(\mathrm{g} / \mathrm{cm}^{3}\right)\end{array}$ & $\begin{array}{l}\text { Radius } \\
R(\mathrm{~cm})\end{array}$ & $\begin{array}{l}\text { Mass } \\
m(\mathrm{~g})\end{array}$ & $\begin{array}{l}v_{.}(\mathrm{m} / \mathrm{sec}) \\
\text { Defined in }(5)\end{array}$ \\
\hline 1 & 0.08 & 1.87 & 2.2 & 8.2 \\
2 & 0.92 & 1.63 & 16.7 & 26.0 \\
3 & 7.70 & 1.65 & 145 & 75.6 \\
4 & 7.70 & $2.47^{\mathrm{a}}$ & 487 & 92.5 \\
5 & 7.70 & $3.14^{\mathrm{a}}$ & 1001 & 104.3 \\
\hline \hline
\end{tabular}

${ }^{a}$ For projectiles 4 and 5 the drag was entirely negligible. They were not spherical; their "radii," defined as $(3 m / 4 \pi \rho){ }^{1 / 3}$, play no role in the analysis and are given here to indicate the size.

$\alpha$ for the values of interest (Fig. 1) allowed us to avoid measuring angles (it was just ensured that the inequality $25^{\circ} \leqslant \alpha \leqslant 50^{\circ}$ held). The thrower would use a projectile five times, to yield a mean value and a typical error (found surprisingly low: 2 or $3 \%$ ). Five projectiles, described in Table I, were launched, and the two unknown parameters $M_{e}$ and $v_{1}$ of the thrower were chosen so as to best fit the five experimental values to the theory. A total of three people repeated the launchings.

According to the theory we have

$$
\frac{g}{v_{*}^{2}}\left(L-\frac{h}{\beta_{f 0}(\alpha, \sigma)}\right) \equiv \frac{g}{v_{*}^{2}} L^{\circ}=\frac{I(\alpha, \sigma)}{\sigma} \text {. }
$$

Here $L^{\circ}$ is the range for zero height of release; $v_{*}^{2}$, defined in (5), depends only on the projectile; and $I$ and $\beta_{f 0}$, defined in (9) and (10), are given in Figs. 1 and 5. We compare theory and experiment in Fig. 6, showing $g L \% v_{*}^{2}$ instead of $g L / v_{*}^{2}$ for compactness. The figure gives the bounds of $I(\alpha, \sigma) / \sigma$ for $25^{\circ} \leqslant \alpha \leqslant 50^{\circ}$. To draw each experimental point an estimate of $h / \beta_{f 0}$ was substracted from the (mean) measured value of $L$. To neglect the effect due to $h$ would lead to a $5 \%-10 \%$ systematic error; on the other hand, though $\beta_{f 0}$ depends noticeably on $\alpha$, and its estimate may err in as much as $30 \%$, it hardly affects the error bounds $(6 \%)$ in $g L \% v_{*}^{2}$, mainly due to a $5 \%$ typical error in the drag coefficient $C_{D}$ in $v_{*}^{2}$. The agreement in Fig. 6 appears to be quite good.

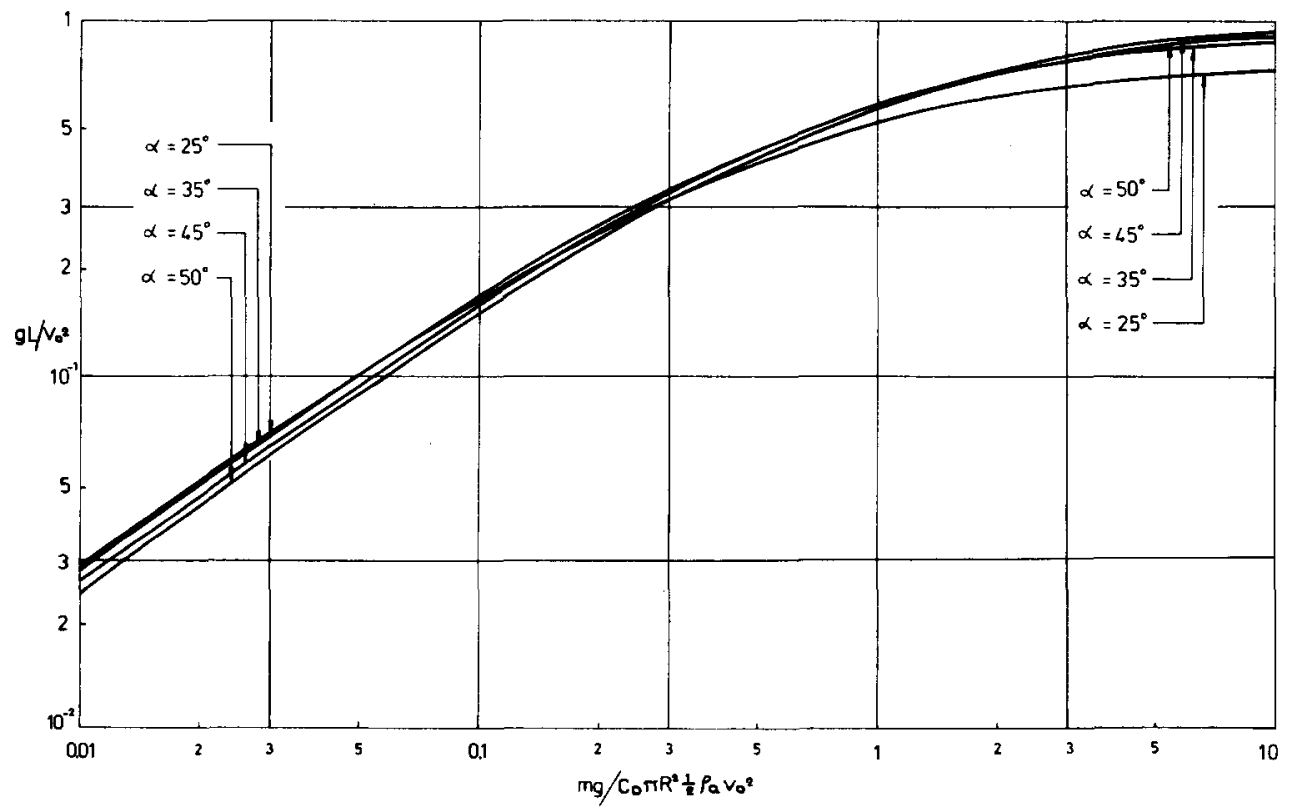

Fig. 6. Dimensionless range (adjusted to zero height of release) versus weight to drag initial ratio; $v_{*}^{2}$, defined in (5). Theory: $a=\alpha_{\mathrm{opt}},(-)$; $25^{\circ}<\alpha<50^{\circ}$, area between lines. Experiment: $\square(\mathrm{a}), O(\mathrm{~b})$, $\Delta$ (c) (see Table II); projectile number of Table I grows from left to right. 
Table II. Thrower characteristics.

\begin{tabular}{|c|c|c|c|c|c|c|}
\hline \multirow[b]{2}{*}{ Thrower } & \multicolumn{3}{|c|}{ Limit velocity $v_{1}(\mathrm{~m} / \mathrm{sec})$} & \multicolumn{3}{|c|}{ Effective mass $M_{e}(\mathrm{~kg})$} \\
\hline & From proj. 1 & From proj. 2 & $\begin{array}{l}\text { Mean value and } \\
\text { error bounds [from (16)] }\end{array}$ & From proj. 4 & From proj. 5 & $\begin{array}{l}\text { Mean value and } \\
\text { error bounds [from (16)] }\end{array}$ \\
\hline$a$ & 26.6 & 25.3 & $26.0 \pm 1.6$ & 0.39 & 0.41 & $0.40 \pm 0.08$ \\
\hline$b$ & 23.7 & 21.7 & $22.7 \pm 1.4$ & 0.45 & 0.53 & $0.49 \pm 0.10$ \\
\hline$c$ & 20.6 & 21.7 & $21.2 \pm 1.3$ & 0.60 & 0.63 & $0.62 \pm 0.12$ \\
\hline
\end{tabular}

To fit theory and experiment for each thrower we first considered projectiles 1 and 2 , for which $v_{0} \simeq v_{L}$. The two different values of $v_{L}$ found in this way, are shown in Table II, together with their mean; also shown are error bounds, obtained independently from (16). Next we considered projectiles 4 and 5 , for which $I \simeq 1$; Table II presents the two values of $M_{e}$ thus found, their mean, and error bounds obtained from (16).

Figure 6 shows that the ratio of ranges by throwers $a$ (having the lowest $M_{e}$ and largest $v_{1}$ ) and $b$ or $c$ is largest for projectile 3 , for which $v_{0} \simeq v_{1}, I \simeq 1$. Although the variation falls within the error bounds, seems to be a real one. In effect, we have

$$
\begin{aligned}
\frac{L_{a 5}}{L_{b 5}} & \simeq \frac{v_{1 a}^{2}}{v_{1 b}^{2}} \frac{1+m_{5} / M_{e b}}{1+m_{5} / M_{e a}}<\frac{v_{1 a}^{2}}{v_{1 b}^{2}} \simeq \frac{L_{a 3}}{L_{b 3}}>\frac{L_{a 1}}{L_{b 1}} \\
& \simeq \frac{I_{\max }\left(v_{*_{1}}^{2} / v_{1 a}^{2}\right)}{I_{\max }\left(v_{*_{1}}^{2} / v_{1 b}^{2}\right)} \frac{v_{1 a}^{2}}{v_{1 b}^{2}} .
\end{aligned}
$$

\section{ACKNOWLEDGMENTS}

We gratefully acknowledge help from J. Meseguer and E. Garmendia in carrying out the measurements.

'S. Timoshenko and D. H. Young, Advanced Dynamics (McGraw-Hill, New York, 1949).

${ }^{2}$ D. B. Lichtenberg and J. G. Willis, Am. J. Phys. 46, 546 (1978).

${ }^{3}$ H. Van Dael and H. Bert, Am. J. Phys. 47, 466 (1979); W. S. Porter, Phys. Teach. 15, 358 (1977).

${ }^{4}$ W. Hooper, Am. J. Phys. 47, 748 (1979).

${ }^{5}$ L. D. Landau and E. M. Lifshitz, Fluid Mechanics (Pergamon, London, 1959).

${ }^{6} \mathrm{R} . \mathrm{McN}$. Alexander and G. Goldspink, Mechanics and Energetics of Animal Locomotion (Chapman and Hall, London, 1977).

\title{
Bohm-Aharonov effect: The quantum mechanics of the electrical transformer
}

\author{
M. Danos \\ National Bureau of Standards, Washington, DC 20234
}

(Received 17 November 1980; accepted for publication 19 March 1981)

A simple physical picture is presented of the Bohm-Aharonov effect.

It seems that the existence of interference in quantum mechanics leads to phenomena that sometimes are in conflict with our preconceived notions. Of course, in that case we must give up the underlying prejudices, a time-consuming task as exemplified by the history of the so-called twin paradox of special relativity. The Bohm-Aharanov effect seems to be of such a nature, and still capable to elicit comments at this late date, half a century after the advent of quantum mechanics (see Ref. 1 , and the references contained therein). It therefore may be worthwhile to describe in as uncomplicated manner as possible the physics behind the Bohm-Aharonov effect. I hope to show that this effect indeed is extremely simple and basic to quantum mechanics. Its connection with the topology of space has been emphasized a long time ago. ${ }^{2}$

Consider the setup of Fig. 1: the electron beam is split into beam 1 and beam 2 by the beam splitter, say a slit; the two beams pass on two sides of the high-permeability iron

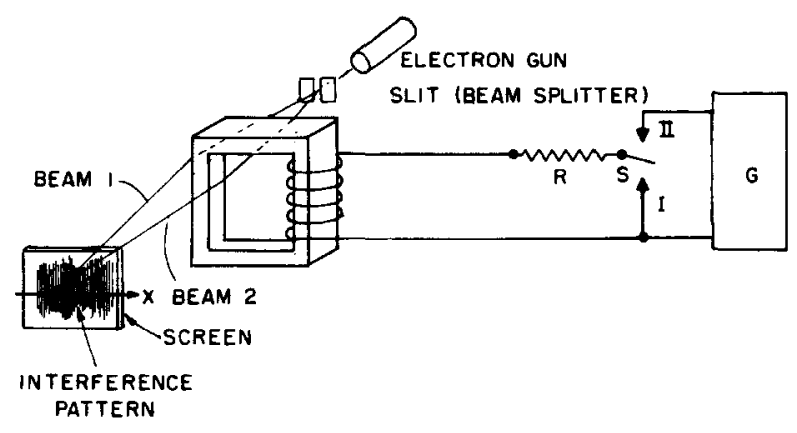

Fig. 1. Schematic picture of the experimental setup. 